\title{
ALTERACIONES MACULARES EN PACIENTES MAYORES DE 50 AÑOS EN UNA UNIDAD DE MEDICINA FAMILIAR
} MACULAR DISORDERS IN PATIENTS OLDER THAN 50 YEARS IN A FAMILYMEDICINE UNIT

Giomara A. Gama-Ortiz ${ }^{1 *}$ y Luz D. Terrazas-Rodríguez ${ }^{2}$

'Unidad de Medicina Familiar N. ${ }^{16}$; 2 Centro de Investigación Educativa y Formación Docente. Instituto Mexicano del Seguro Social, Querétaro, México

RESUMEN: Antecedentes: Las alteraciones maculares representadas principalmente por la degeneración macular asociada a la edad son causa de ceguera en mayores de 50 años. Objetivo: Determinar la prevalencia de alteraciones maculares en pacientes mayores de 50 años. Material y métodos: Estudio transversal descriptivo durante el periodo enero-febrero del 2020 en pacientes mayores de 50 años, mediante muestreo no probabilístico por conveniencia. Muestra calculada mediante fórmula de porcentajes para población infinita con nivel de confianza del $95 \%$, obteniendo 280 pacientes. Las alteraciones maculares se detectaron utilizando la prueba de la rejilla de Amsler. El análisis estadístico incluyó promedios, porcentajes e intervalos de confianza. Resultados: Promedio de edad 66.4 años, el $37.5 \%$ del grupo etario 61 a 70 años, sexo femenino un $60 \%$, escolaridad primaria el $36.4 \%$ y dedicadas al hogar el 39.3\%. El 50.7\% no fumadores, con hipertensión arterial el $56.8 \%$, con diabetes tipo 2 el $37.9 \%$ y con diagnóstico de una sola patología el $44.6 \%$. El $10.4 \%$ con alteraciones maculares en ojo izquierdo, un $8.2 \%$ con alteraciones maculares ojo derecho; solo un ojo alterado el $16.4 \%$ y ambos ojos el $1.1 \%$. Conclusión: La prevalencia de alteraciones maculares es del $17.5 \%$, lo que incrementa el riesgo de alguna patología que derive en ceguera.

Palabras clave: Prevalencia. Macular. Rejilla Amsler.
ABSTRACT: Background: Macular alterations represented mainly by age-related macular degeneration are the cause of blindness in people over 50 years of age. Objective: To determine the prevalence of macular alterations in patients older than 50 years. Material and methods: Descriptive cross-sectional study during the period January-February 2020 in patients over 50 years of age, using non-probabilistic convenience sampling. Sample calculated using the percentage formula for an infinite population with a confidence level of $95 \%$, obtaining 280 patients. Macular changes were detected using the Amsler grid test. Statistical analysis included means, percentages, and confidence intervals. Results: Average age 66.4 years; age group 61 to 70 years $(37.5 \%)$; female sex $(60 \%)$ primary schooling $(36.4 \%)$ and dedicated to the home $(39.3 \%)$. The $(50.7 \%)$ non-smokers; arterial hypertension (56.8\%); type 2 diabetes (37.9\%); diagnosis of a single pathology (44.6\%). Macular alterations in the left eye $(10.4 \%)$; right eye $(8.2 \%)$; only one altered eye (16.4\%) and both eyes (1.1\%). Conclusion: The prevalence of macular alterations is $17.5 \%$, which increases the risk of some pathology that leads to blindness.

Key words: Prevalence. Macular. Amsler grid.
Correspondencia:

*Giomara A. Gama-Ortiz

E-mail: gamaortiz90@gmail.com
Fecha de recepción: 19-11-2020

Fecha de aceptación: 25-02-2021
Disponible en internet: 05-05-2021

Rev Mex Med Fam. 2021;8:15-20 DOI: 10.24875/RMF.20000183

2007-9710 / @ 2021 Federación Mexicana de Especialistas y Residentes en Medicina Familiar. Publicado por Permanyer. Este es un artículo open access bajo la licencia CC BY-NC-ND (http://creativecommons.org/licenses/by-nc-nd/4.0/). 


\section{INTRODUCCIÓN}

En el mundo existen alrededor de 314 millones de personas con discapacidad visual, de las cuales 45 millones padecen ceguera. La incidencia de enfermedades infecciosas ha disminuido, sin embargo, debido al aumento de la esperanza de vida se ha incrementado la prevalencia de enfermedades cronicodegenerativas. Aproximadamente un $82 \%$ de las personas con discapacidad visual son mayores de 50 años, pero solo representan un 19\% de la población mundial. El número de personas en riesgo de sufrir discapacidad visual relacionada con la edad incrementa en relación con el aumento de la población mundial y la proporción de adultos mayores ${ }^{1,2}$.

La tendencia de la pirámide poblacional hacia el envejecimiento es clara, lo que aumentará la demanda de servicios de salud por enfermedades crónicas y sus consecuencias, una de ellas son las alteraciones maculares, representadas principalmente por la degeneración macular asociada a la edad (DMAE), que son causa del 7\% de ceguera en personas mayores de 50 años y representan el $3 \%$ de la deficiencia visual en el mundo ${ }^{1,2}$.

La mácula tiene síntomas muy característicos, ya que cualquier alteración anatómica o vascular produce distorsión o pérdida de la visión central; por ello sus manifestaciones son tempranas y notables. Los síntomas patognomónicos de trastornos maculares son las metamorfopsias (anormalidades de la forma de las cosas) y menos específicos, pero igualmente orientadores son la disminución de agudeza visual, la visión borrosa y los escotomas centrales ${ }^{3,4}$.

La rejilla de Amsler fue inicialmente introducida en 1947 por el médico suizo Marc Amsler, inspirado en el diseño original de Landolt, quien describió en 1847 un dispositivo que consistía en una serie de líneas paralelas con un centro de fijación utilizado para determinar la presencia o ausencia de una metamorfopsia ${ }^{5}$.

Debido a su sencillez y objetividad, la prueba de función macular mediante la cartilla de Amsler es obligada en la evaluación de pacientes sospechosos de enfermedad macular. La prueba se efectúa haciendo mirar al paciente, con cada ojo por separado, una cuadrícula blanca con un punto en el centro. Se pide al sujeto que dibuje o diga si la cuadrícula presenta cualquier irregularidad, alteración o escotomas ${ }^{3}$.

Existen diferentes patologías que pueden afectar al área macular, conocidas en conjunto como «síndrome macular», las cuales comparten la característica de afectar la visión central; principalmente son: membrana epirretiniana, agujero macular, DMAE y edema macular ${ }^{6,7}$.

Se estima que el número de personas que padecen DMAE se incrementó en un 50\% en el $2020^{8}$. Por lo tanto, el diagnóstico precoz y la realización del tratamiento es esencial para ralentizar la progresión y la pérdida de la visión posterior, detectar a tiempo las complicaciones y lograr una mejor adaptación a la enfermedad'.

En este contexto, el objetivo del artículo es determinar la prevalencia de alteraciones maculares en pacientes mayores de 50 años en una unidad de medicina familiar.

\section{METODOLOGÍA}

Se realizó un estudio transversal descriptivo, en pacientes mayores de 50 años, en la Unidad de Medicina Familiar N. ${ }^{\circ} 16$ del Instituto Mexicano del Seguro Social, delegación Querétaro, en el periodo comprendido entre enero y febrero del 2020.

Se incluyeron pacientes que aceptaron participar en el estudio previo consentimiento informado, con edad igual o mayor a 50 años, hombres y mujeres. Excluyéndose pacientes con ceguera total, con diagnóstico previo de alguna alteración macular y pacientes con problemas de refracción que no contaban en el momento de la entrevista con su auxiliar visual para la lectura de la rejilla de Amsler.

Se elaboró un muestreo no probabilístico, por conveniencia, donde el tamaño de muestra $(n=280)$ se calculó con la fórmula de porcentajes para población infinita con nivel de confianza del 95\% $(\mathrm{z}$ alfa $=1.64)$, potencia de la prueba del $80 \%(\mathrm{z}$ beta $=0.84)$, prevalencia de alteraciones maculares del 13\% ( $\mathrm{p}=0.13)$ y margen de error del $5 \%(d=0.05)$.

Se utilizó un formato de encuesta para los datos sociodemográficos, los cuales fueron edad, sexo, escolaridad y ocupación, si contaban con diagnóstico de hipertensión 
Tabla 1. Características sociodemográficas

\begin{tabular}{|c|c|c|c|c|}
\hline \multirow[t]{2}{*}{ Característica } & \multirow[t]{2}{*}{ Frecuencia } & \multirow[t]{2}{*}{ Porcentaje } & \multicolumn{2}{|c|}{ IC 95\% } \\
\hline & & & Inferior & Superior \\
\hline \multicolumn{5}{|l|}{ Sexo } \\
\hline Femenino & 168 & 60.0 & 54.0 & 65.0 \\
\hline Masculino & 112 & 40.0 & 34.0 & 45.0 \\
\hline \multicolumn{5}{|l|}{ Escolaridad } \\
\hline Sabe leer y escribir & 23 & 8.2 & 4.9 & 11.0 \\
\hline Primaria & 102 & 36.4 & 30.0 & 42.0 \\
\hline Secundaria & 60 & 21.4 & 16.5 & 26.2 \\
\hline Preparatoria & 45 & 16.1 & 11.7 & 20.4 \\
\hline Universidad & 50 & 17.9 & 13.3 & 22.3 \\
\hline \multicolumn{5}{|l|}{ Ocupación } \\
\hline Ama de casa & 110 & 39.3 & 33.5 & 45.0 \\
\hline Obrero & 4 & 1.4 & 0.0 & 2.0 \\
\hline Empleado & 50 & 17.9 & 13.3 & 22.3 \\
\hline Profesionista & 4 & 1.4 & 0.0 & 2.0 \\
\hline Comerciante & 10 & 3.6 & 1.3 & 5.7 \\
\hline Jubilado & 90 & 32.1 & 26.6 & 37.6 \\
\hline Otra & 12 & 4.3 & 1.9 & 6.6 \\
\hline
\end{tabular}

IC: intervalo de confianza.

arterial y/o diabetes mellitus independientemente de si estuvieran en control o no, y si tenían antecedente de tabaquismo.

Para la valoración de las alteraciones maculares se utilizó la prueba de la rejilla de Amsler, la cual se realizó monocularmente, primero en el ojo derecho y después en el ojo izquierdo utilizando un oclusor ocular. Como primer paso se le indicó al paciente permanecer sentado, se colocó la rejilla de Amsler a 20-30 cm de distancia frente al ojo a explorar, cada ojo tuvo que estar compensado ópticamente de así requerirlo para la distancia de la prueba.

Para evaluar el campo visual central se pidió al paciente que mantuviera la mirada en el punto central de la rejilla y se le realizaron las siguientes preguntas: ¿Puede ver un punto negro en el centro de la cuadrícula? Manteniendo la mirada en el punto negro ¿puede ver las cuatro esquinas del cuadrado grande? ¿Puede ver los cuatro lados del cuadrado? ¿Ve todos los cuadritos intactos, o ve algún agujero, interrupción o ausencia en las líneas? ¿Aparecen borrosas en algún lugar?
¿Dónde? (señalar sin tocar la lámina). ¿Ve todas las líneas rectas y paralelas? ¿Son todos los cuadritos del mismo tamaño? ¿Ve alguna cosa más, movimiento de las líneas, vibraciones, algo brillante o algún color? En caso afirmativo, ¿puede indicar dónde? ¿Ve alguna parte de la cuadrícula borrada? ¿Cuántos cuadritos hay entre el centro y el borramiento?

Por último, en función de las respuestas obtenidas se determinó si el campo visual central se encontraba normal o alterado, normal si no respondía positivo respecto a alguna alteración y alterado si respondía positivo a una sola alteración del campo visual. De igual forma se repitió el procedimiento en el ojo contralateral.

El análisis se realizó con la ayuda del paquete estadístico SPSS, versión 25, e incluyó promedios, intervalos de confianza para promedios, porcentajes e intervalos de confianza para porcentajes.

Este estudio fue valorado y aprobado por el comité local de investigación y ética, y siguió en todo momento las normas establecidas en la declaración de Helsinki para 
Tabla 2. Características clínicas

\begin{tabular}{|c|c|c|c|c|}
\hline \multirow[t]{2}{*}{ Característica } & \multirow[t]{2}{*}{ Frecuencia } & \multirow[t]{2}{*}{ Porcentaje } & \multicolumn{2}{|c|}{ IC 95\% } \\
\hline & & & Inferior & Superior \\
\hline \multicolumn{5}{|l|}{ Tabaquismo } \\
\hline Fumador & 28 & 10.0 & 6.4 & 13.5 \\
\hline Fumador pasivo & 32 & 11.4 & 7.6 & 15.1 \\
\hline Exfumador & 78 & 27.9 & 22.5 & 33.1 \\
\hline No fumador & 142 & 50.7 & 44.8 & 56.6 \\
\hline \multicolumn{5}{|c|}{ Presencia de diagnóstico de hipertensión arterial } \\
\hline Sí & 159 & 56.8 & 50.9 & 62.6 \\
\hline No & 121 & 43.2 & 37.3 & 49.0 \\
\hline \multicolumn{5}{|l|}{ Presencia de diagnóstico de diabetes tipo 2} \\
\hline Sí & 106 & 37.9 & 32.1 & 43.5 \\
\hline No & 174 & 62.1 & 56.4 & 67.8 \\
\hline \multicolumn{5}{|c|}{ Distribución de acuerdo con la combinación de morbilidad } \\
\hline Diabetes mellitus e hipertensión arterial & 70 & 25.0 & 20.0 & 30.0 \\
\hline Diabetes o hipertensión & 125 & 44.6 & 38.1 & 50.0 \\
\hline Ninguna patología & 85 & 30.4 & 25.0 & 35.3 \\
\hline
\end{tabular}

reforzar la protección y los beneficios de los participantes en la investigación, temas relacionados con el registro de información y el consentimiento sobre la investigación.

\section{RESULTADOS}

De los 280 pacientes que conformaron la muestra, 168 (60\%) fueron mujeres y 112 $(40 \%)$ hombres. En relación con la edad, se tuvo un mínimo de 50 y un máximo de 94 años, el promedio de edad de los pacientes encuestados fue 66.4 años (intervalo de confianza del 95\% [IC 95\%]: 56.7-76.1), con mayor presencia del grupo etario de 61 a 70 años (37.5\%; IC 95\%: 31.5-43.0), predominaron el sexo femenino (60\%; IC 95\%: 54.0-65.0), la escolaridad primaria (36.4\%; IC 95\%: 30.0 42.0) y como empleo, las dedicadas al hogar (39.3\%; IC 95\%: 33.5-45.0) (Tabla 1).

El 50.7\% (IC 95\%: 44.8-56.6) de los entrevistados no eran fumadores; la comorbilidad que prevaleció fue la hipertensión arterial (56.8\%; IC 95\%: 50.9-62.6), seguida de la diabetes mellitus (37.9\%; IC 95\%: 32.143.5), de las cuales no se obtuvo información sobre si estaban en control o no; el $44.6 \%$ (IC 95\%: 38.1-50.0) presentaba diagnóstico de una sola patología. La tabla 2 presenta el resto de la información.

El ojo que presentaba un mayor predominio de alteraciones maculares fue el ojo izquierdo (10.4\%; IC 95\%: 6.7-13.9), seguido del ojo derecho (8.2\%; IC 95\%: 4.9-11.5); con presencia de un solo ojo alterado un $16.4 \%$ (IC 95\%: 11.7-20.2) y el 1.1\% presentaba alteración en ambos ojos (IC 95\%: -0.0 a 2.2). Véase la tabla 3 para el resto de la información.

\section{DISCUSIÓN}

El estudio de las alteraciones maculares es una patología pocas veces estudiada, ya que en el primer nivel de atención no se realiza evaluación visual de forma sistemática, no obstante, la importancia del trabajo que aquí se presenta estriba en el conocimiento generado en torno a la detección; tema de importancia en el actual contexto de los sistemas de salud involucrados, ya que sin tratamiento se desarrolla pérdida importante de la visión en el ojo afectado, en un periodo de dos años. La detección oportuna de alteraciones maculares desde un primer nivel de atención se ha relacionado con una menor cantidad de complicaciones visuales con ayuda de un método sencillo y accesible como lo es la rejilla de Amsler, ${ }^{9,10}$. 
Tabla 3. Prevalencia de alteraciones maculares

\begin{tabular}{|c|c|c|c|c|}
\hline \multirow[t]{2}{*}{ Alteraciones maculares } & \multirow[t]{2}{*}{ Frecuencia } & \multirow[t]{2}{*}{ Porcentaje } & \multicolumn{2}{|c|}{ IC 95\% } \\
\hline & & & Inferior & Superior \\
\hline \multicolumn{5}{|l|}{ 0jo derecho } \\
\hline Normal & 257 & 91.8 & 88.5 & 95.0 \\
\hline Alterado & 23 & 8.2 & 4.9 & 11.5 \\
\hline \multicolumn{5}{|l|}{ 0jo izquierdo } \\
\hline Normal & 251 & 89.6 & 86.0 & 93.2 \\
\hline Alterado & 29 & 10.4 & 6.7 & 13.9 \\
\hline \multicolumn{5}{|c|}{ Distribución de alteraciones maculares } \\
\hline Ambos ojos normales & 231 & 82.5 & 77.5 & 86.4 \\
\hline Un ojo alterado & 46 & 16.4 & 11.7 & 20.2 \\
\hline Dos ojos alterados & 3 & 1.1 & -0.0 & 2.2 \\
\hline
\end{tabular}

Para la evaluación de las alteraciones maculares se utilizó la rejilla de Amsler. Es un método de detección portátil, accesible, de bajo costo y con una razonable especificidad y sensibilidad, que se utiliza para determinar la presencia o ausencia de una metamorfopsia, tomando el resultado de acuerdo con la prueba en normal o alterado. A causa de su objetividad y sencillez, la prueba de función macular mediante la rejilla de Amsler en pacientes sospechosos de patología macular es obligada, sin embargo, no debe considerarse por sí sola una prueba de confirmación en el diagnóstico, ya que es necesaria la evaluación integral oftalmológica utilizando equipo especializado. Desafortunadamente, por diversos factores llevar a cabo esta última implica un mayor tiempo de espera para el paciente, por lo que se propone, como médicos de primer nivel de atención, que se deben utilizar los instrumentos y herramientas que están a nuestro alcance con la finalidad de realizar a tiempo una pesquisa y evitar así desenlaces fatales ${ }^{1,3,5,11,12}$.

En cuanto a las características sociodemográficas de la población estudiada, se encuentra que la media de edad es similar a la reportada en la literatura ${ }^{13}$. Con respecto al sexo, con predominio del femenino, observándose el mismo patrón con relación al sexo reportado por Hernández-Narváez (2015), que fue del $70.1 \%{ }^{13}$, y diferente a lo reportado por Delgado (2016), donde predominó el sexo masculino con un $52.5 \%{ }^{14}$. La razón de esto se puede encontrar en el hecho de que a la clínica elegida para tomar la muestra acude un mayor porcentaje de mujeres, otro punto a considerar es el área geográfica en la cual fue aplicada.

El nivel escolar de los pacientes es bajo, al respecto se debe entender que se trata de una población que nació en la década de los años 50 o 60 del siglo pasado, momento del tiempo caracterizado por el acceso limitado al sistema educativo.

La mayor parte de la población encuestada fueron mujeres, dedicadas al hogar. No se encontraron estudios con estas mismas características sociodemográficas para poder realizar una comparativa con el presente, sin embargo, la explicación se puede encontrar en el cambio de rol posterior a la jubilación, en este caso el papel de la mujer a nivel doméstico la lleva a desempeñar el rol de cuidadora y encargada de las labores de hogar.

En cuanto al tabaquismo, prevalecieron pacientes no fumadores, afirmación contraria a lo reportado a la literatura ${ }^{15}$. La explicación de lo anterior puede ligarse al hecho de que en este estudio fueron mayormente mujeres las encuestadas.

En relación con las comorbilidades, en el presente trabajo prevalecen enfermedades crónico degenerativas como la hipertensión arterial, seguida de la diabetes mellitus, similar a lo reportado en la literatura ${ }^{13}$. La explicación se puede encontrar en que estadísticamente hay una mayor cantidad de pacientes con diagnóstico de hipertensión arterial. 
$\mathrm{Al}$ analizar las alteraciones maculares, el ojo que presenta mayor predominio de alteraciones maculares es el ojo izquierdo, seguido del ojo derecho; con predominio de un solo ojo alterado. La prevalencia de alteraciones maculares en el presente estudio es mayor a lo reportado en otros estudios ${ }^{13,14}$. Estas diferencias se deben a la forma de evaluación visual: en este análisis se utilizó únicamente como instrumento la rejilla de Amsler aplicada para un primer nivel de atención, mientras que los otros estudios mencionados fueron realizados por oftalmólogos con equipo especializado a pacientes con alguna patología visual; por otra parte, el tamaño de muestra del presente fue menor en comparación con los otros.

\section{CONCLUSIONES}

En la población estudiada se detectó una prevalencia de alteraciones maculares del $17.5 \%$, con predominio en el grupo de 61 a 70 años con un $6 \%$, seguido del grupo de 71 a 80 años con un $5.7 \%$, y por último el grupo de 50 a 60 años con un $2.8 \%$. Se observa una media de edad con mayor daño macular de $65.94 \pm 2.7$ años con predominio del sexo femenino (58.69\%). Con respecto a las enfermedades crónico degenerativas, se identificó mayor presencia de alteraciones maculares en pacientes con hipertensión arterial (63.95\%). En relación con el tabaquismo, no se encontró asociación, ya que predominaron los pacientes no fumadores.

Con base en los resultados obtenidos se propone que el médico familiar haga énfasis en realizar acciones preventivas y en la detección oportuna, para evitar así la progresión de la enfermedad, derivando al servicio de oftalmología a tiempo con el objetivo de disminuir el riesgo de padecer ceguera, deterioro del funcionamiento diario e impacto negativo en la calidad de vida del paciente, así como evitar el aumento en los costos de la atención médica.

\section{FINANCIAMIENTO}

Los autores no recibieron patrocinio para llevar a cabo este artículo.

\section{CONFLICTO DE INTERESES}

No existe conflicto de intereses por parte de los autores.

\section{RESPONSABILIDADESÉTICAS}

Protección de personas y animales. Los autores declaran que para esta investigación no se han realizado experimentos en seres humanos ni en animales.

Confidencialidad de los datos. Los autores declaran que han seguido los protocolos de su centro de trabajo sobre la publicación de datos de pacientes.

Derecho a la privacidad y consentimiento informado. Los autores declaran que en este artículo no aparecen datos de pacientes.

\section{BIBLIOGRAFÍA}

1. Guía de práctica clínica. Diagnóstico y tratamiento de la degeneración macular relacionada con la edad [Internet]. México; Instituto Mexicano del Seguro Social; 2010. Disponible en: http://www. cenetec.salud.gob.mx/descargas/gpc/CatalogoMaestro/401 IMSS_10_Deg_Macular_x_edad/EyR_IMSS_401_10.pdf

2. Miqueli M, López S, Rodríguez S. Baja visión y envejecimiento de la población. Rev Cuba Oftalmol. 2016;29(3):492-501.

3. Quiroz M. La mácula y su patología. En: Graue Wiechers E, editor. Oftalmología en la práctica de la medicina general. 4. ${ }^{\text {a }}$ edición. México, D.F.: Mc Graw-Hill Interamericana; 2014. pp. 211-221.

4. Sánchez J. Maculopatías. En: Meyran García J., editor. Criterios clínicos, diagnósticos y terapéuticos en Oftalmología. 1. ${ }^{\text {a edi- }}$ ción. México, D.F.: Intersistemas; 2004. pp. 61-64.

5. Hernández L. Retina y vítreo. En: Rojas S, Saucedo A, editores. Técnicas de exploración del polo posterior. 2. ${ }^{a}$ edición. Ciudad de México, México: El Manual Moderno; 2012. pp. 49-50.

6. Patología macular [Internet]. Madrid, España: Sociedad Española de Oftalmología; junio de 2018. Disponible en: https://www.oftalmoseo.com/patologias-frecuentes-2/patologia-macular/.

7. Guía de práctica clínica. Diagnóstico y tratamiento del agujero macular idiopático [Internet]. México: Instituto Mexicano del Seguro Social; 2013. Disponible en: http://www.imss.gob.mx/sites/all/statics/guiasclinicas/614GRR.pdf.

8. González AE. La calidad de vida relacionada con la salud en pacientes con degeneración macular asociada a la edad [trabajo de fin de máster en Internet] [Santander]: Universidad de Cantabria; septiembre de 2017. Disponible en: http://repositorio.unican.es:8080/xmlui/bitstream/handle/10902/12878/gonzalezalvarezeva.pdf?sequence=4\&isallowed=y.

9. Fraccaro $P$, Nicolo $M$, Bonetto $M$, Giacomini M, Weller P, Enrico $C$, et al. Combining macula clinical signs and patient characteristics for age-related macular degeneration diagnosis: a machine learning approach. BMC Ophthalmol. 2015;15:10.

10. Kankanahalli S, Burlina PM, Wolfson Y, Freund DE, Bressler NM Clasificación automatizada de la gravedad de la degeneración macular relacionada con la edad a partir de fotografías de fondo de ojo. IOVS. 2013;54(3):1789-96.

11. Rodríguez Calderón V. Actualización de las pruebas para el diagnóstico clínico y la detección precoz de la DMAE [trabajo de fin de máster en Internet]. [Alicante]: Universidad de Alicante; 2014. Disponible en: http://hdl.handle.net/10045/40162.

12. Amsler M. Earliest symptoms of diseases of the macula. Brit J Ophthal. 1953;37:521-37.

13. Hernández-Narváez MG, Olivares-Luna AM, Carillo-Hernández A, Tovar-Méndez GM, González-Pedraza A. Prevalence of visual disorders and their relationship with functionality of the older adults. Rev Cubana Oftalmol. 2015;28(2):190-7.

14. Delgado Chávez MC. Correlación entre la rejilla de Amsler y la tomografía de coherencia óptica en la degeneración macular asociada a la edad, en pacientes de 60 a 85 años en el club social cultural deportivo [trabajo de fin de máster en Internet]. [Quito]: Tecnológico Superior Cordillera; 2016. Disponible en: http://www.dspace.cordillera.edu.ec:8080/xmlui/handle/1234 56789/2014.

15. Quintero M, Perea C, Padilla C, Rojas I, Rodríguez S, Luis I. Capacidad funcional y calidad de vida en los ancianos con degeneración macular y baja visión. Rev Cubana Oftalmol. 2014;27(3):332-49. 